\title{
EchoGéo
}

$53 \mid 2020$

Dénominations plurielles. Quand les noms de lieux se concurrencent

\section{Des mots pour le dire}

Jean Louis Chaléard

\section{CpenEdition}

Journals

Édition électronique

URL : https://journals.openedition.org/echogeo/20158

DOI : 10.4000/echogeo.20158

ISSN : 1963-1197

Éditeur

Pôle de recherche pour l'organisation et la diffusion de l'information géographique (CNRS UMR 8586)

Référence électronique

Jean Louis Chaléard, « Des mots pour le dire », EchoGéo [En ligne], 53 | 2020, mis en ligne le 25 octobre 2020, consulté le 11 août 2021. URL : http://journals.openedition.org/echogeo/20158 ; DOI : https:// doi.org/10.4000/echogeo.20158

Ce document a été généré automatiquement le 11 août 2021.

EchoGéo est mis à disposition selon les termes de la licence Creative Commons Attribution - Pas d'Utilisation Commerciale - Pas de Modification 4.0 International (CC BY-NC-ND) 


\title{
Des mots pour le dire
}

\author{
Jean Louis Chaléard
}

1 Le numéro 53 d'Échogéo est largement consacré à la toponymie, objet d'un volumineux dossier Sur le champ. Le texte qui porte sur un album Panini dans la rubrique Sur le métier y fait aussi plusieurs fois allusion. C'est donc sous le signe des appellations géographiques, des mots pour désigner les lieux, de leur sens explicite, implicite ou caché, qu'est tournée la présente livraison de notre revue. La toponymie a des liens avec de nombreuses disciplines : l'histoire, l'anthropologie, la sociologie, l'économie, la géopolitique... Mais comme étude des noms propres désignant des lieux, elle concerne peut-être plus encore la géographie.

2 Le dossier de la rubrique Sur le champ est centré sur les « dénominations plurielles » et met en évidence les dimensions multiples des concurrences entre noms de lieux. Frédéric Giraut, qui l'a dirigé, observe dans son introduction que les pluralités d'appellations, éventuellement contradictoires et fonctionnant sur des registres différents, sont fréquentes. Elles sont révélatrices de représentations et de pratiques mais aussi de rapports historiques et de tensions politiques et/ou sociales. Au-delà de la grande diversité des cas analysés, de la Première Guerre mondiale à l'époque contemporaine, des États-Unis ou de la France à l'Afrique et au Népal, des métropoles au monde rural, les articles du dossier mettent en évidence la richesse des registres vernaculaires et l'importance des enjeux politiques ou sociétaux que révèlent les querelles de mots ou les dénominations multiples.

Quelques contributions prennent en compte fortement les dimensions patrimoniale et géopolitique des noms de lieux. Deux s'intéressent à la guerre de 1914-1918 qui a amené une nouvelle couche d'appellations se superposant, de façon pérenne ou temporaire, à un fond rural. Alain Devos, Pierre Taborelli et Robin Perarnau, étudiant sur les champs de bataille de la Grande Guerre en Champagne les noms des tranchées et des boyaux, soulignent l'appropriation du terrain par les combattants. Celle-ci se manifeste par une répartition territoriale des noms de tranchées à l'échelle locale autour de thèmes diversifiés, renforçant la valeur identitaire du groupe producteur. Dans son étude sur la Grande Guerre à travers la gestion des odonymes (noms des routes et places) en France, Clément Millon essaie de mesurer la mémoire vivante du passé. Cette dernière paraît 
importante, attestant de la présence des guerres dans le quotidien des Français, mais moins que celle de la Seconde Guerre mondiale ce qui amène l'auteur à s'interroger sur ce fait et sur les conséquences de l'éloignement progressif dans le temps de la Grande Guerre.

4 Avec le texte de Ghousmane Mohamed nous sommes encore dans la question du patrimoine. L'auteur s'intéressant à l'onomastique touareg, aux portes du Sahara nigérien, montre en quoi la toponymie touareg révèle un système socio-spatial cohérent et un environnement interprété selon ses usages et les circulations d'une société mobile. La création toponymique/onomastique est un des lieux privilégiés de la mémoire collective touareg. À une autre échelle, Christophe Gauchon s'intéresse également aux processus de patrimonialisation en étudiant la production de toponymes à partir de la dénomination des biens inscrits sur la liste du Patrimoine mondial de l'Unesco. L'inscription sur cette liste est l'occasion de réinventions toponymiques qui consacrent, aux côtés des appellations entérinées par l'usage, des noms expressément construits à des fins de patrimonialisation. Le discours du patrimoine apparaît alors comme un des moyens par lesquels se déploie la politique culturelle des États. Darshan Karki et Miriam Wenner prolongent la réflexion sur la dimension politico-identitaire de la néotoponymie en analysant l'ambivalence des noms de lieux dans des plaines népalaises. Ils cherchent à comprendre comment les élites utilisent les toponymes, soit pour appuyer les revendications territoriales et l'appartenance à la nation, soit pour saper ces mêmes revendications. Ils mettent en lumière ainsi les contradictions des processus de dénomination en place dans ce pays où ceux-ci se mêlent à la politique ethnique et à la restructuration territoriale.

Dans la toponymie vernaculaire s'expriment, de façon variable, des formes réelles de contestation ou de résilience. Jean-Baptiste Bing à partir des exemples parisiens et morvandiaux analyse comment la toponymie véhiculée par les contes et légendes, l'oraliture, permet de promouvoir ou maintenir une toponymie et un imaginaire vernaculaires, ancrés dans le passé, et vient promouvoir des usages des lieux alternatifs à des projets ou des opérations d'aménagement. La ville africaine informelle est le siège d'une production toponymique importante qui reflète les clivages au sein de la société urbaine entre le pouvoir politique et les habitants des quartiers populaires. Gaston Ndock Ndock met en évidence les oppositions, à Yaoundé, dans les opérations de régularisation urbaine entre des noms de lieux officiels que l'administration tente d'imposer et les noms vernaculaires qui restent en usage. Dorcas Zuvalinyenga, dans une ville moyenne du Zimbabwe, Bindura, examine la coexistence en couches superposées et l'utilisation simultanée d'une gamme complexe de toponymes. Il montre que l'utilisation de différents noms pour un même lieu met en lumière les tensions et les négociations en cours entre les diverses identités dans la ville, sur des bases variées : historiques, sociales, culturelles, etc. Les noms et les pratiques de nommage d'un lieu nous fournissent, dans ce cas comme dans d'autres, des éclairages sur son passé et sur les comportements de ses habitants. Loin de l'Afrique, à Boston, Elsa Vivant se penche sur les différences toponymiques dans un secteur urbain à l'interface de trois quartiers où la vente, la consommation de drogue et la prise en charge des usagers sont pratiquées de façons contrastées. Les réactions et demandes des riverains de ces différents quartiers sont révélatrices des rapports de pouvoir dans l'espace urbain dont les choix toponymiques sont les analyseurs. Comme dans les cas précédents, mais dans 
un tout autre contexte, la toponymie vernaculaire révèle les tensions liées aux problèmes et au fonctionnement de la société urbaine.

6 Fabio Armand et Jean-Pierre Gerfaud étudient les tautologies (dénominations dont la forme condense deux expressions transmettant un seul et même concept) en toponymie, en se concentrant sur les noms composés. Ils montrent que ces derniers apparaissent avec un changement dans le peuplement et le statut des langues qui entraine une perte de sens du concept associé au mot. Le nom ancien est conservé, mais complété par une référence au concept dans la langue du dominateur. L'analyse toponymique permet ici de repérer les enjeux de continuité ou de fracture qui se jouent dans des sociétés aux langues, cultures et situations politiques différentes. Au total, le dossier insiste sur ce que la pluralité des appellations révèle de poids des héritages, de ruptures, de pratiques et de tensions sociales ou politiques. Surtout, comme le souligne Frédéric Giraut dans son introduction, il met en évidence la richesse et la puissance politique des registres vernaculaires lorsqu'ils sont en concurrence avec des registres officiels.

7 Dans le texte de la rubrique Sur le métier il est aussi question de toponymie, même si ce n'est pas l'essentiel du sujet, et de ses relations au patrimoine. Xavier Leroux, professeur des écoles, se propose d'évaluer l'intérêt d'une expérience pédagogique auprès des élèves de quatre classes de l'enseignement primaire : l'utilisation de l'album Panini «collectionne Tourcoing", chez un éditeur célèbre pour ses vignettes d'animaux et surtout de footballeurs. C'est l'occasion pour les élèves de chercher à identifier les lieux de la ville représentés sur les images. À travers cette expérience l'auteur interroge l'album en lui-même (pertinence de la photographie, du toponyme retenu pour l'illustrer) mais également le rapport des élèves à la pratique de l'espace.

8 C'est à une réflexion sur la photographie, sur ses relations avec les sciences sociales et une dénonciation des effets des agropesticides que nous propose le texte de Benjamin Lysaniuk, Anaïs Ondet et Léa Prost dans la rubrique Sur l'image. L'exploitation de deux clichés, un portrait et un paysage, tirés de la série documentaire "Les mauvaises herbes » d'Anaïs Ondet, offre l'occasion de questionner les notions d'invisibilité, de mémoire, de reconnaissance et de mettre dans la lumière des victimes que l'on n'entend en général pas et des paysages qu'on ne remarque plus. Comme l'observent les auteurs, cette contribution met en évidence la fécondité d'un dialogue entre des démarches artistiques et scientifiques visant à révéler des processus sociaux et économiques liés à des décisions politiques.

Bien sûr, comme la plupart des activités du pays, la publication des numéros d'Échogéo est contrainte par la présence de la covid-19 et les mesures prises pour l'endiguer. Audelà des problèmes quotidiens, nous essayons d'apporter notre éclairage sur la situation actuelle de la pandémie et ses conséquences. Dans le numéro précédent, une contribution sur Ho Chi Minh Ville et les deux textes de la rubrique Sur le métier s'en faisaient l'écho. Le dernier article de la rubrique Sur le vif traite d'un volet important et discuté, en France comme à l'étranger : le confinement. Rémi de Bercegol, Anthony Goreau-Ponceaud, Shankare Gowda et Antony Ra abordent la situation dans les quartiers marginalisés en Inde. La mise en place d'un confinement en mars 2020 a provoqué une forte panique parmi les populations déshéritées fuyant désespérément les grandes villes pour retourner chez elles. Mais ceux qui n'avaient pas d'autres choix sont restés confinés dans les marges urbaines. Les auteurs soulignent les conditions de vie très dures de ces derniers et les effets paradoxaux d'un confinement non adapté aux 
quartiers pauvres. En France, la presse s'est faite l'écho d'enquêtes de l'Inserm qui montrent que la covid-19 est une maladie socialement (et spatialement) inégalitaire. Même si les situations sont difficilement comparables, une certaine convergence des observations pousse à interroger d'autres cas pour voir ce qui relève du particulier et ce qui témoigne d'évolutions générales. Nous serons amenés à revenir sur ces questions... 